\section{REVIEW ARTICLE}

\author{
Michael Bölker* \\ Tel: + 496421 2821536. Fax: + 496421 2828971. e-mail: boelker@mailer.uni-marburg.de
}

\title{
Ustilago maydis - a valuable model system for the study of fungal dimorphism and virulence
}

Universität Marburg, Fachbereich Biologie, Karl-von-Frisch-Strasse 8, D-35032 Marburg, Germany

Keywords: pathogenicity, plant pathogen, cAMP signalling, mating, MAP kinase signalling

\section{Overview}

The dimorphic phytopathogenic fungus Ustilago maydis already has a long tradition in fungal biology and genetics (Brefeld, 1883; Christensen, 1963; Holliday, 1974). It has been used to study the molecular basis of such diverse phenomena as mating type determination, homologous recombination and signalling pathways. In this review, I will concentrate on the potential of $U$. maydis to serve as a model organism for the study of fungal dimorphism and pathogenesis. When compared to other systems, $U$. maydis exhibits a number of genetic properties that make it an excellent candidate to elucidate the molecular mechanisms of these complex phenomena. U. maydis cells can exist both as budding cells and as true hyphae. Cells of the widely used fungal model system Saccharomyces cerevisiae are able to switch only between budding and a pseudohyphal type of growth. During the life cycle of $U$. maydis, nonpathogenic haploid cells, which show yeast-like growth, have to fuse to form dikaryotic hyphae, which are then able to infect maize plants. The dimorphic switch and the transition between the nonpathogenic and pathogenic lifestyle are regulated by a single master control locus that encodes a pair of transcription factors. This has allowed the construction of a pathogenic haploid strain that can be used in genetic screens to search for mutants which are affected in virulence. In addition, genes that have been identified in reverse genetic approaches can be tested in this genetic background. Generation of knockout mutants is very easy due to effective homologous recombination. Diploid strains of $U$. maydis can be used to maintain mutant alleles of essential genes in their heterozygous state.

This favourable situation has allowed diverse approaches to identify components of the regulatory network that controls dimorphism and virulence in $U$. maydis. The ultimate goal is the understanding of pathogenic development at all levels of genetic regulation and biochemical action. U. maydis has been the subject of a number of reviews. A comprehensive overview, which covers the early literature, was given by
Christensen (1963). More recent reviews focus on the molecular mechanism of mating type determination (Banuett, 1995; Kahmann \& Bölker, 1996) and the importance of signalling pathways during mating and pathogenesis (Banuett, 1998; Kahmann et al., 1999; Kronstad et al., 1998; Lengeler et al., 2000).

\section{Life cycle of $U$. maydis}

U. maydis is a hemibasidiomycete and has a very narrow host range. It infects only maize (Zea mays) and its progenitor plant teosinte (Zea mays subsp. parviglumis). Symptoms of the disease are the formation of large

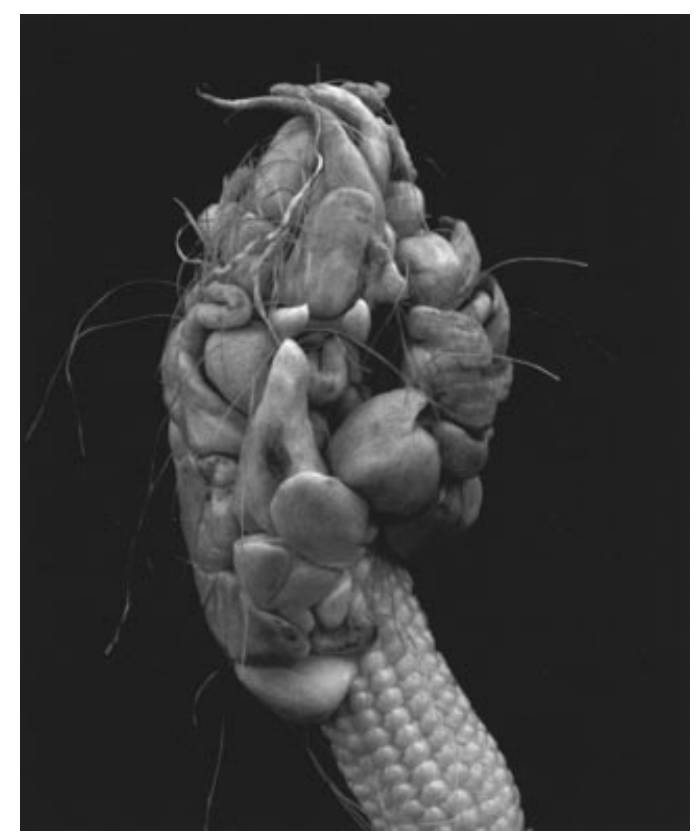

Fig. 1. Tumours on the cob of a maize plant induced by $U$. maydis. Infected kernels have grown to considerable size. Within the tumour tissue, proliferation of fungal mycelium and spore formation occurs. 


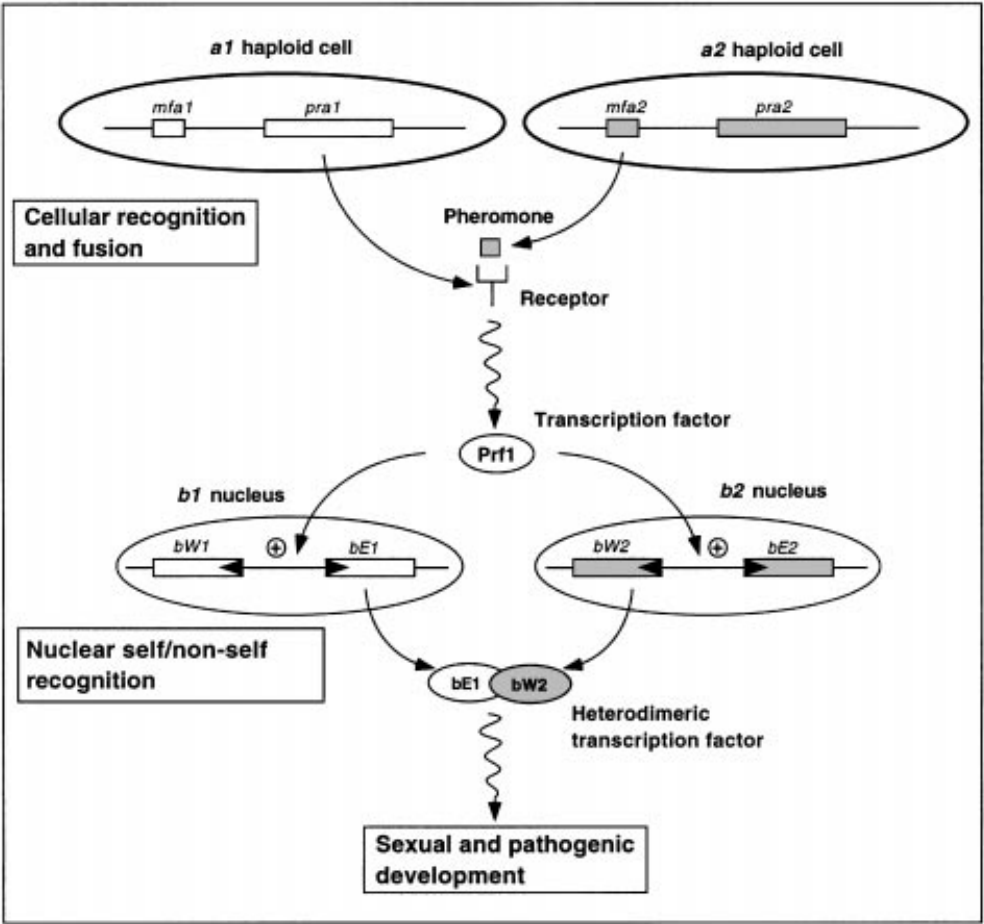

Fig. 2. Interactions between the two mating-type loci during development of $U$. maydis. The biallelic a locus encodes a pheromone-based cell recognition system, which triggers cell fusion. After fusion, different alleles at the multiallelic $b$ locus induce sexual and pathogenic development by formation of an active heterodimeric transcription factor.

tumours (or galls), which can develop on all green parts of the plant, including stems, leaves, tassels and ears. The fungal mycelium proliferates within these tumours, which can reach a remarkable size (see Fig. 1). Pathogenic development is completed by the release of large quantities of highly melanized, diploid teliospores. These spores are distributed by air and can germinate under favourable conditions. During germination, meiosis occurs and results in the production of haploid, unicellular sporidia.

In nature, pathogenic and sexual development of $U$. maydis is inseparably connected. Haploid cells multiply vegetatively by budding and are saprophytic; they are unable to infect plants. Fusion of compatible haploid cells is required to generate a dikaryon, which grows in a hyphal form and which is strictly dependent on the host plant for sustained growth. Completion of the sexual cycle and spore production occurs under normal circumstances only in the infected plant. A long-standing goal of Ustilago researchers has been reached quite recently, when Ruiz-Herrera et al. (1999) reported the successful production of sexual spores in fungal mycelium grown in close contact with embryogenic maize cell cultures in vitro. This will open a new window to follow the molecular details of fungal development during plant infection.

The most conspicuous symptom of the disease caused by $U$. maydis is the formation of plant tumours by induction of cell proliferation in meristematic plant tissue (Fig. 1). It was always tempting to speculate that phytohormones produced by the fungus are responsible for this reaction (Wolf, 1952). This hypothesis was strongly supported by the observation that in liquid cultures the fungus releases significant amounts of the plant hormone auxin (indole3-acetic acid) (Wolf, 1952). In addition, infected tumour tissue exhibits a 5- to 20-fold elevated concentration of auxin. As yet, however, it is unclear whether this auxin is produced by the fungus or by the plant as a response to the infection. Recently, an attempt has been made to abolish auxin production of $U$. maydis by disrupting the gene for an indole-3-acetaldehyde dehydrogenase suspected to be involved in the biosynthetic pathway (Basse et al., 1996). However, in the absence of glucose as a carbon source, the resulting strain was still able to produce auxin, indicating the existence of an additional indole-3-acetaldehyde dehydrogenase whose expression might be subject to catabolite repression. Consequently, these mutant strains were not affected in pathogenicity and tumour induction (Basse et al., 1996). Thus, no conclusive evidence for a crucial role of fungus-derived phytohormone production during pathogenic development has yet been achieved.

\section{Genetic control of mating and pathogenesis in $U$. maydis}

The fusion of cells and the development of the pathogenic dikaryon are governed by two independent genetic loci that function as a mating type determination system (see Fig. 2). The biallelic a locus encodes precursors for specific lipopeptide pheromones and for receptors that can sense the presence of pheromones of opposite mating type (Bölker et al., 1992). Fusion of haploid cells occurs only between cells that differ at the a locus, i.e. an a1 cell can only fuse with an a2 cell. During mating, cells secrete mating-type-specific lipopeptide pheromones 
and respond to the presence of cells of opposite mating type (Spellig et al., 1994). As a consequence of pheromone recognition, cells arrest in their cell cycle and form conjugation tubes that orient their growth toward the pheromone source (Snetselaar et al., 1996). These mating hyphae eventually fuse at their tips. Both nuclei migrate into the common cytoplasm and form a dikaryotic cell.

At this stage, a genetic self versus non-self recognition reaction between the two nuclei occurs (Fig. 2). Only if they contain different alleles of the multiallelic $b$ locus is a stable dikaryon formed, which grows as a long straight filament and is able to infect corn plants. The $b$ locus contains a pair of divergently transcribed genes, $b \mathrm{~W}$ and $b E$, which both code for putative transcription factors of the homeodomain family (Gillissen et al., 1992; Kronstad \& Leong, 1990; Schulz et al., 1990). The existence of more than 25 different $b$ alleles implies that many hundreds of possible combinations of $b$ alleles, for example, $b 12$ and $b 22$ or $b 4$ and $b 17$, all result in filament formation and pathogenicity (Kahmann \& Bölker, 1996). Cells carrying different $a$ alleles but identical $b$ alleles will fuse but do not form a stable dikaryon and are not pathogenic for maize plants. Elegant genetic and biochemical experiments have elucidated the molecular mechanism of this multiallelic self/non-self recognition system. The $\mathrm{bW}$ and $\mathrm{bE}$ homeodomain proteins can form heterodimers, but only if they are derived from different alleles; for example, $\mathrm{bW} 1$ protein is able to interact with bE2, bE3, bE4 etc. but not with bE1 (Kämper et al., 1995). The same is true for all other combinations of different $\mathrm{bW}$ and $\mathrm{bE}$ proteins. A highly variable stretch of about 100 amino acids at the N-terminus of both proteins is responsible for this allele-specific recognition system (Kämper et al., 1995). The $\mathrm{bW} / \mathrm{bE}$ heterodimer is thought to act as a transcriptional activator or repressor that ultimately regulates the expression of genes required for the pathogenic reaction of the dikaryon (Fig. 2). Thus, in $U$. maydis a single master regulatory locus determines whether the organism is virulent or not. A haploid strain has been constructed that carries a chimeric $b$ locus consisting of a $b W$ and a $b E$ allele derived from different mating types. Although this strain differs from normal haploid wild-type strains only in a single gene, it is able to infect maize plants and to complete the sexual life cycle (Bölker et al., 1995b). This haploid pathogenic strain has been used in genetic screens for mutants that are affected in pathogenicity.

\section{Signalling affects morphology and virulence in $U$. maydis}

During the infection process, environmental and hostspecific cues are perceived and transmitted by intracellular signalling pathways. Thus it was not surprising to notice that virulence of $U$. maydis is severely reduced if components of such transduction pathways are disrupted. However, closer inspection revealed an interesting cross-talk between cAMP signalling and a

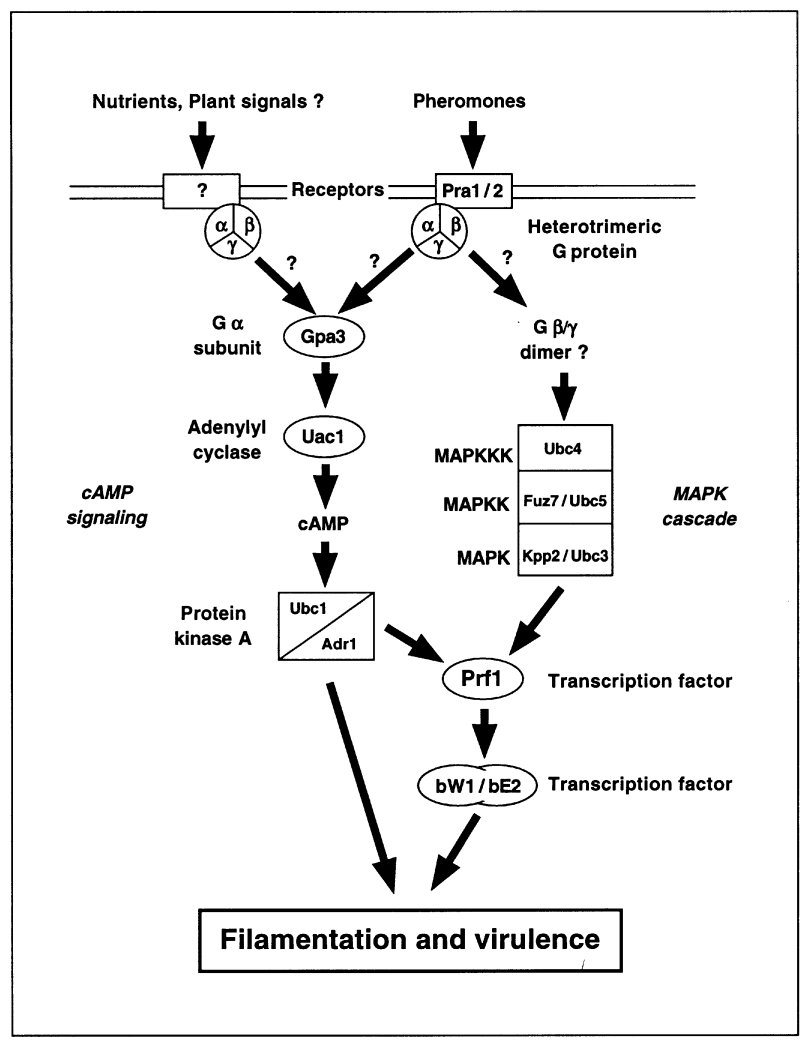

Fig. 3. Schematic overview of the interplay between CAMP signalling and the pheromone response pathway during pathogenic development. Integration of these different regulatory pathways occurs at different levels and results in the induction of filamentation and virulence.

MAP kinase (MAPK) cascade (see Fig. 3). The starting point of these investigations was the analysis of the pheromone response reaction and the observation that mutants affected in cAMP signalling displayed an altered cell morphology (Gold et al., 1994). The pheromone response is elicited by stimulation of receptors belonging to the family of G-protein-coupled seven-transmembrane proteins. Four different genes encoding G protein $\alpha$ subunits, gpa1 to gpa4, were identified. Whereas deletion of gpa3 resulted in sterility and avirulence, the remaining three $G \alpha$ subunits are apparently not involved in mating and pathogenicity (Regenfelder et al., 1997). Surprisingly, gpa3 is also required for virulence of pathogenic strains, which carry a chimeric $b$ allele and thus are not dependent on cell fusion for initiating pathogenic development. This has prompted a more detailed study of this $\mathrm{G} \alpha$ subunit. It was demonstrated that Gpa3 belongs to the subfamily of adenylylcyclase-stimulating $\alpha$ subunits and that gpa3 mutants exhibit elongated cell morphology. The morphological phenotype and the mating defect could be suppressed by addition of exogenous cAMP (Krüger et al., 1998). These observations point to a cross-talk between the pheromone response pathway and cAMP, but put into question the direct role of Gpa3 in coupling to the pheromone receptor. 
The role of cAMP signalling in regulating dimorphism in U. maydis has been analysed in detail in the groups of J. Kronstad and S. Gold. The gene for adenylylcyclase, uac1, was identified in a screen for mutants exhibiting a constitutive filamentous phenotype (Barrett et al., 1993; Gold et al., 1994). The subsequent isolation of extragenic suppressors for $u a c 1$ led to the identification of $u b c 1$, which encodes the regulatory subunit of cAMP-dependent protein kinase A (PKA). It was demonstrated that low levels of intracellular cAMP result in elongated cells, whereas high levels of cAMP or the constitutive activation of PKA activity in the absence of the regulatory subunit induce a multiple budding phenotype (Gold et al., 1994). Two genes, uka1 and adr1, encoding potential catalytic subunits of PKA were identified by sequence homology. Whereas deletion of $u k a 1$ did not result in an obvious mating defect, $a d r 1$ mutants exhibit the same filamentous phenotype as uac1 mutants and are epistatic to $u b c 1$ (Dürrenberger \& Kronstad, 1999; Dürrenberger et al., 1998). This indicates that a functional cAMP signalling pathway is required for nutritional sensing, mating and pathogenic development.

Surprisingly, additional suppressors of nac1 turned out to be members of a MAP kinase (MAPK) signalling cascade, with Ubc3 acting as a MAPK, Ubc5 as a MAPKK and Ubc4 as a MAPKKK (Andrews et al., 2000; Mayorga \& Gold, 1998, 1999). Interestingly, two of these components have also been identified as members of the pheromone signalling pathway. Ubc5 is identical to Fuz7 (Banuett \& Herskowitz, 1994), which has been suggested to be involved in pheromone signalling, and Ubc3 is identical to Kpp2, which has been identified as MAPK, being part of the pheromone response pathway (Müller et al., 1999).

The cAMP and MAPK pathways involved in environmental and pheromone signalling appear to be connected at two levels. The first level is a $G \propto \alpha$ subunit, Gpa3, which was originally identified as part of the pheromone response pathway but which is apparently also involved in cAMP signalling. Its essential function for mating could be replaced by the addition of exogenous cAMP (Krüger et al., 1998). This could indicate that either Gpa3 is not directly coupled to the pheromone receptors or that Gpa3 is coupled to multiple receptors, perhaps in combination with different $\beta / \gamma$ heterodimers. If one assumes that activation of the MAPK kinase cascade occurs by the $\beta / \gamma$ heterodimer, stimulation of the pheromone receptors could result in activation of both the cAMP and the MAPK signalling pathway (Fig. 3). To explain why gpa3 mutant cells cannot mate if exogenous cAMP is added, one has to assume that cell fusion may require the activated receptor for directing the growth of conjugation tubes and the spatial co-ordination of membrane fusion events. This could indicate that either the $\beta / \gamma$ heterodimer triggers these events or that the receptor itself interacts with components of the cell fusion apparatus.

The second level of cross-talk between cAMP and MAPK signalling occurs at the pivotal transcription factor Prf1, which regulates the pheromone-induced expression of the $a$ and $b$ mating type genes. The Prf1 protein carries several sequence motifs specific for PKAand MAPK-dependent phosphorylation and the presence of these sites is essential for Prf1 function (Hartmann et al., 1999). In addition, expression of the prf1 gene itself is subject to nutritional stimulation and to positive feedback control after pheromone stimulation (Hartmann et al., 1999). One has to assume that basal activity of Prf1 requires phosphorylation by lowlevel constitutive activity of the MAPK Kpp2/Ubc3. Nutritional signalling results in stimulation of Prf1 activity at two different levels, the induction of expression and additional phosphorylation of PKA sites. The fact that members of the MAPK cascade have been identified as suppressors of mutants lacking adenylylcyclase indicates that the basal level of MAPK phosphorylation of Prf1 (or another transcription factor) is required for the filamentous growth of cells lacking cAMP.

The existence of pathogenic haploid strains indicates that recognition of pheromones is not essential during infection. Nevertheless, both the cAMP signalling pathway and the MAPK cascade are required for virulence. This has been taken as evidence that these signalling networks are also engaged in sensing specific plant signals that trigger discrete stages of pathogenic development. This was supported by the observation that expression of a constitutive active variant of the $\mathrm{G} \propto$ subunit, $\mathrm{Gpa} 3_{\mathrm{Q} 260 \mathrm{~L}}$, still allows tumour induction but interferes with fungal proliferation and spore production (Krüger et al., 2000).

The genetic dissection of signal transduction cascades in $U$. maydis has added another fascinating piece to the growing puzzle of these networks and their function in fungal development and virulence. Although similar elements occur in a number of different fungi, their specific functions appear to vary significantly between these species. A comprehensive overview of this topic has been given recently by Lengeler et al. (2000).

\section{Identification of virulence genes in $U$. maydis}

The ability of pathogenic organisms to infect their hosts has often been ascribed to specific 'virulence genes'. At a first approximation, these genes can be defined as those that are required for the pathogenic process. Genetically, this would mean that deletion of these genes would result in a loss of virulence.

However, not every gene whose deletion results in attenuated virulence necessarily plays a specific role during this stage. Many enzymes involved in the biosynthesis of amino acids and nucleotides are not required on complete media. During propagation inside the host, genes for such biosynthetic enzymes may become essential since amino acids and nucleotides are not, or at least not in sufficient amounts, provided by the host. Thus, many such viable mutants, which are reduced in their general fitness, may also be unable to 
infect the host plant. Mutations leading to loss of virulence are therefore not necessarily informative about functions specifically involved in pathogenic development. Discrimination between whether such a candidate gene can be regarded as a specific virulence gene, or as just a fitness gene, is rather difficult.

On the other hand, genes that do play important roles during infection processes are often present in several copies. Fungal species that attack plants or animals often contain batteries of genes encoding isoenzymes involved in, for example, cell-wall degradation or proteolysis (Hube, 1996; Koller et al., 1995). Disruption of one, or even several, of these genes does not necessarily reduce virulence. Thus, in a genetic screen for avirulent mutants this important set of pathogenicity genes may escape detection. These genes may be identified by their specific expression patterns. Many different methods to identify infection-specific genes of pathogenic organisms have been described, such as differential display of RNA, cDNA-amplified fragment length polymorphism (cDNA-AFLP) and in vivo expression technology (IVET) (for a review, see Hensel \& Holden, 1996).

\section{Generation of avirulent mutants by plasmid insertion}

Haploid pathogenic U. maydis strains have been constructed that allow an unbiased search for mutants affected in virulence. Mutants can easily be produced by UV or chemical mutagenesis and scored for their ability to induce tumours in maize plants. The identification of the affected genes, however, is very tedious because it requires positional cloning or complementation of the mutant phenotype by transformation with a cosmid library. To avoid these laborious procedures, plasmid insertion mutagenesis has been established for U. maydis (Bölker et al., 1995a). Transformation of protoplasts with DNA in the presence of restriction enzymes results in preferential insertion of the incoming DNA into genomic restriction sites (for a recent review, see Maier $\&$ Schäfer, 1999). If the insertion disrupts an ORF which is essential for the pathogenic process, this should result in loss of virulence. Transformants are screened in a pathogenicity test and potential candidates, which are nonpathogenic, can be identified. The affected gene can easily be isolated by cloning the inserted plasmid DNA with flanking sequences (Bölker et al., 1995a). To confirm that the observed phenotype is indeed caused by the plasmid insertion, an identical mutation can be constructed by replacing the wild-type copy with the tagged version by homologous recombination. But even if disruption of a gene clearly results in loss of virulence, it is not an easy task to define whether this gene exerts a specific function during the infection process or whether it has only a role in general fitness. Indeed, a considerable number of mutants have been identified that are avirulent, but none of the affected genes has yet been attributed to a clearly defined function during pathogenic development.

\section{Isolation of pathogenicity genes by their specific expression pattern}

In addition, isolation of pathogenicity genes of $U$. maydis has been attempted by studying their specific expression patterns. Those functions that are required for pathogenic development are expected to be induced upon entry into the host. By subtractive hybridization, the filament-specific gene egl1 was identified, which encodes an endoglucanase. Disruption of the genomic copy, however, did not result in avirulence (Schauwecker et al., 1995). Isolation of a highly abundant hyphal cell-wall component led to identification of a gene, rep1, which is expressed only during filamentous growth (Wösten et al., 1996). It encodes secreted small proteins that contribute to the hydrophobicity of the fungal cell wall. Another gene, mig1, which is highly expressed during growth in the plant, was identified by differential display (Basse et al., 2000). From its derived amino acid sequence it was concluded that mig1 encodes a small, highly charged protein of unknown function which contains a functional $\mathrm{N}$ terminal secretion sequence. Again, deletion of this gene did not result in loss of virulence (Basse et al., 2000). The promoter of this gene has been analysed for regulatory elements that are responsible for the expression pattern. It has been shown that this gene is subject to both negative and positive control (Basse et al., 2000). The detailed analysis of additional genes that exhibit a similar expression pattern will help to elucidate common regulatory elements involved in the activation of hostinduced genes.

\section{The existence of a single master control locus for pathogenicity allows a top-down approach}

A specific feature of $U$. maydis is the existence of a single master control locus that determines both mating type and pathogenicity. This allows a specific approach to the study of the molecular mechanisms of fungal pathogenicity that is distinct to those used in most other systems. The introduction of an active allele at the single $b$ locus in a haploid strain is sufficient to transform a nonpathogenic cell into a pathogenic one (Bölker et al., $1995 \mathrm{~b})$. Thus, the complex process of pathogenic development is solely controlled by this gene locus. In principle, it should be possible to identify downstream components of this regulatory cascade all the way down to the level of genes that are directly involved in the pathogenic process.

The $b$ gene products belong to the family of homeodomain transcription factors and thus are believed to be either activators or repressors of other specific promoters (Gillissen et al., 1992; Schulz et al., 1990). Until recently, all attempts to identify a direct DNA target for the active $\mathrm{bW} / \mathrm{bE}$ protein complex have been unsuccessful. At least one target has now been isolated by the combination of genetic and biochemical approaches: transcription of the genes encoding phero- 
mone precursors and pheromone receptors is induced upon recognition of pheromone of opposite mating type (Urban et al., 1996b). After cell fusion, expression is turned down to a low, but constitutive level due to the presence of the active $\mathrm{bW} / \mathrm{bE}$ heterodimer. Within the a2 mating type allele an ORF, lga2, exists, to which a biological function has not yet been assigned (Urban et al., 1996a). Expression of lga2 is not only induced upon pheromone stimulation, but is even further increased after cell fusion has occurred. The expression of $\lg a 2$ is also enhanced in cells that carry two different alleles of the $b$ locus (Urban et al., 1996b). Thus, this gene is a prime candidate for direct regulation through the $\mathrm{bW} / \mathrm{bE}$ homeodomain complex. It has been successfully used to identify a short DNA sequence in its promoter region that is required for $b$-dependent regulation. In vitro DNA binding studies clearly demonstrated direct recognition of this sequence by a chimeric $\mathrm{bW} / \mathrm{bE}$ fusion protein (Romeis et al., 2000). However, lga2 cannot be the only target of the $\mathrm{bW} / \mathrm{bE}$ heterodimer since it is not required for pathogenicity. The identification of a functional binding site for the $\mathrm{bW} / \mathrm{bE}$ heterodimer will clearly help to study the interaction of the many different $\mathrm{bE} / \mathrm{bW}$ protein complexes with DNA in more detail. In addition, this finding might lead to the isolation of further promoter sequences which contain similar sequence elements.

To identify genes that act further downstream of the master regulator, a screen has been performed for mutants that allow the expression of dikaryon-specific genes, for example the endoglucanase egll, in the absence of an active bW-bE heterodimer. By this approach a gene, rum1, was identified which encodes a polypeptide highly similar to the human retinoblastoma binding protein 2 (Quadbeck-Seeger et al., 2000). From its domain structure Rum1 is supposed to interact with histone deacetylases. Histone deacetylases act as corepressors and decrease the concentration of acetylated histone, which correlates with actively transcribed DNA regions ( $\mathrm{Ng} \& \mathrm{Bird}, 2000)$. This indicates that the switch from the nonpathogenic to the pathogenic phase might be accompanied by a rearrangement of the heterochromatin structure. It is still unclear how this alteration of chromatin structure may be induced by the $\mathrm{bW} / \mathrm{bE}$ heterodimer, which is supposed to act as a transcriptional regulator. In addition, it would be interesting to know which set of genes is affected by this global regulation and how these genes are involved in the control of pathogenicity.

\section{Outlook}

The genetic control of mating, dimorphism and pathogenicity during the life cycle of $U$. maydis has been characterized in molecular detail over the last 10 years. Regulation of morphogenesis and virulence involves a complex network of regulatory and signalling components. In contrast to many other plant-pathogenic fungi, $U$. maydis is very amenable to classical and molecular genetic analysis and has the advantage of existing in a haploid saprophytic stage, which allows efficient genetic manipulation. In addition, U. maydis is the subject of two privately funded genome projects (Bayer AG, Germany, and Exelixis, Inc., USA). U. maydis is thus the first member of the Basidiomycete group of fungi whose entire genomic sequence is known. Modern genomic and postgenomic techniques such as high-density DNA microarrays now allow biologists to study the many facets of the parasitic fungal-plant interaction. The parallel analysis of expression levels of thousands of genes during different stages of hostpathogen interactions will certainly help to identify candidate virulence genes whose expression is specifically regulated during pathogenic development. The availability of these modern experimental techniques for future research places $U$. maydis on the growing list of eukaryotic model organisms.

\section{References}

Andrews, D. L., Egan, J. D., Mayorga, M. E. \& Gold, S. E. (2000). The Ustilago maydis $u b c 4$ and $u b c 5$ genes encode members of a MAP kinase cascade required for filamentous growth. Mol Plant-Microbe Interact 13, 781-786.

Banuett, F. (1995). Genetics of Ustilago maydis, a fungal pathogen that induces tumours in maize. Annu Rev Genet 29, 179-208.

Banuett, F. (1998). Signalling in the yeasts: an informational cascade with links to the filamentous fungi. Microbiol Mol Biol Rev 62, 249-274.

Banuett, F. \& Herskowitz, I. (1994). Identification of fuz7, a Ustilago maydis MEK/MAPKK homolog required for $a$-locusdependent and -independent steps in the fungal life cycle. Genes Dev 8, 1367-1378.

Barrett, K. J., Gold, S. E. \& Kronstad, J. W. (1993). Identification and complementation of a mutation to constitutive filamentous growth in Ustilago maydis. Mol Plant-Microbe Interact 6, 274-283.

Basse, C. W., Lottspeich, F., Steglich, W. \& Kahmann, R. (1996). Two potential indole-3-acetaldehyde dehydrogenases in the phytopathogenic fungus Ustilago maydis. Eur J Biochem 242, 648-656.

Basse, C. W., Stumpferl, S. \& Kahmann, R. (2000). Characterization of a Ustilago maydis gene specifically induced during the biotrophic phase: evidence for negative as well as positive regulation. Mol Cell Biol 20, 329-339.

Bölker, M., Urban, M. \& Kahmann, R. (1992). The a mating type locus of U. maydis specifies cell signaling components. Cell 68, 441-450.

Bölker, M., Böhnert, H. U., Braun, K. H., Görl, J. \& Kahmann, R. (1995a). Tagging pathogenicity genes in Ustilago maydis by restriction enzyme-mediated integration (REMI). Mol Gen Genet 248, 547-552.

Bölker, M., Genin, S., Lehmler, C. \& Kahmann, R. (1995b). Genetic regulation of mating and dimorphism in Ustilago maydis. Can J Bot 73, S320-S325.

Brefeld, O. (1883). Botanische Untersuchungen über Hefenpilze. Untersuchungen aus dem Gesammtgebiete der Mykologie V. Die Brandpilze. Leipzig.

Christensen, J. J. (1963). Corn Smut Caused by Ustilago maydis. Monograph no. 2. St Paul, MN: American Phytopathological Society.

Dürrenberger, F. \& Kronstad, J. (1999). The $u k c 1$ gene encodes a protein kinase involved in morphogenesis, pathogenicity and 
pigment formation in Ustilago maydis. Mol Gen Genet 261, 281-289.

Dürrenberger, F., Wong, K. \& Kronstad, J. W. (1998). Identification of a cAMP-dependent protein kinase catalytic subunit required for virulence and morphogenesis in Ustilago maydis. Proc Natl Acad Sci US A 95, 5684-5689.

Gillissen, B., Bergemann, J., Sandmann, C., Schroeer, B., Bölker, M. \& Kahmann, R. (1992). A two-component regulatory system for self/non-self recognition in Ustilago maydis. Cell 68, 647-657.

Gold, S., Duncan, G., Barrett, K. \& Kronstad, J. (1994). cAMP regulates morphogenesis in the fungal pathogen Ustilago maydis. Genes Dev 8, 2805-2816.

Hartmann, H. A., Krüger, J., Lottspeich, F. \& Kahmann, R. (1999). Environmental signals controlling sexual development of the corn smut fungus Ustilago maydis through the transcriptional regulator Prf1. Plant Cell 11, 1293-1306.

Hensel, M. \& Holden, D. W. (1996). Molecular genetic approaches for the study of virulence in both pathogenic bacteria and fungi. Microbiology 142, 1049-1058.

Holliday, R. (1974). Ustilago maydis. In Handbook of Genetics, pp. 575-595. Edited by R. C. King. New York: Plenum.

Hube, B. (1996). Candida albicans secreted aspartyl proteinases. Curr Top Med Mycol 7, 55-69.

Kahmann, R. \& Bölker, M. (1996). Self/nonself recognition in fungi: old mysteries and simple solutions. Cell 85, 145-148.

Kahmann, R., Basse, C. \& Feldbrügge, M. (1999). Fungal-plant signalling in the Ustilago maydis-maize pathosystem. Curr Opin Microbiol 2, 647-650.

Kämper, J., Reichmann, M., Romeis, T., Bölker, M. \& Kahmann, R. (1995). Multiallelic recognition: nonself-dependent dimerization of the $\mathrm{bE}$ and $\mathrm{bW}$ homeodomain proteins in Ustilago maydis. Cell $81,73-83$.

Koller, W., Yao, C., Trial, F. \& Parker, D. M. (1995). Role of cutinase in the invasion of plants. Can J Bot 73, S1109-S1118.

Kronstad, J. W. \& Leong, S. A. (1990). The $b$ mating-type locus of Ustilago maydis contains variable and constant regions. Genes Dev 4, 1384-1395.

Kronstad, J., De Maria, A. D., Funnell, D., Laidlaw, R. D., Lee, N., de Sa, M. M. \& Ramesh, M. (1998). Signaling via cAMP in fungi: interconnections with mitogen-activated protein kinase pathways. Arch Microbiol 170, 395-404.

Krüger, J., Loubradou, G., Regenfelder, E., Hartmann, A. \& Kahmann, R. (1998). Crosstalk between cAMP and pheromone signalling pathways in Ustilago maydis. Mol Gen Genet 260, 193-198.

Krüger, J., Loubradou, G., Wanner, G., Regenfelder, E., Feldbrügge, M. \& Kahmann, R. (2000). Activation of the cAMP pathway in Ustilago maydis reduces fungal proliferation and teliospore formation in plant tumours. Mol Plant-Microbe Interact 13, 1034-1040.

Lengeler, K. B., Davidson, R. C., D'Souza, C., Harashima, T., Shen, W. C., Wang, P., Pan, X., Waugh, M. \& Heitman, J. (2000). Signal transduction cascades regulating fungal development and virulence. Microbiol Mol Biol Rev 64, 746-785.

Maier, F. J. \& Schäfer, W. (1999). Mutagenesis via insertional- or restriction enzyme-mediated-integration (REMI) as a tool to tag pathogenicity related genes in plant pathogenic fungi. Biol Chem 380, 855-864.

Mayorga, M. E. \& Gold, S. E. (1998). Characterization and molecular genetic complementation of mutants affecting dimorphism in the fungus Ustilago maydis. Fungal Genet Biol 24, 364-376.

Mayorga, M. E. \& Gold, S. E. (1999). A MAP kinase encoded by the $u b c 3$ gene of Ustilago maydis is required for filamentous growth and full virulence. Mol Microbiol 34, 485-497.

Müller, P., Aichinger, C., Feldbrügge, M. \& Kahmann, R. (1999). The MAP kinase kpp2 regulates mating and pathogenic development in Ustilago maydis. Mol Microbiol 34, 1007-1017.

Ng, H. H. \& Bird, A. (2000). Histone deacetylases: silencers for hire. Trends Biochem Sci 25, 121-126.

Quadbeck-Seeger, C., Wanner, G., Huber, S., Kahmann, R. \& Kämper, J. (2000). A protein with similarity to the human retinoblastoma binding protein 2 acts specifically as a repressor for genes regulated by the $b$ mating type locus in Ustilago maydis. Mol Microbiol 38, 154-166.

Regenfelder, E., Spellig, T., Hartmann, A., Lauenstein, S., Bölker, M. \& Kahmann, R. (1997). G proteins in Ustilago maydis: transmission of multiple signals? EMBO J 16, 1934-1942.

Romeis, T., Brachmann, A., Kahmann, R. \& Kämper, J. (2000). Identification of a target gene for the bE-bW homeodomain protein complex in Ustilago maydis. Mol Microbiol 37, 1-14.

Ruiz-Herrera, J., Leon-Ramirez, C., Cabrera-Ponce, J. L., MartinezEspinoza, A. D. \& Herrera-Estrella, L. (1999). Completion of the sexual cycle and demonstration of genetic recombination in Ustilago maydis in vitro. Mol Gen Genet 262, 468-472.

Schauwecker, F., Wanner, G. \& Kahmann, R. (1995). Filamentspecific expression of a cellulase gene in the dimorphic fungus Ustilago maydis. Biol Chem Hoppe-Seyler 376, 617-625.

Schulz, B., Banuett, F., Dahl, M., Schlesinger, R., Schäfer, W., Martin, T., Herskowitz, I. \& Kahmann, R. (1990). The $b$ alleles of U. maydis, whose combinations program pathogenic development, code for polypeptides containing a homeodomain-related motif. Cell 60, 295-306.

Snetselaar, K. M., Bölker, M. \& Kahmann, R. (1996). Ustilago maydis mating hyphae orient their growth toward pheromone sources. Fungal Genet Biol 20, 299-312.

Spellig, T., Bölker, M., Lottspeich, F., Frank, R. W. \& Kahmann, R. (1994). Pheromones trigger filamentous growth in Ustilago maydis. EMBO J 13, 1620-1627.

Urban, M., Kahmann, R. \& Bölker, M. (1996a). The biallelic a mating type locus of Ustilago maydis: remnants of an additional pheromone gene indicate evolution from a multiallelic ancestor. Mol Gen Genet 250, 414-420.

Urban, M., Kahmann, R. \& Bölker, M. (1996b). Identification of the pheromone response element in Ustilago maydis. Mol Gen Genet 251, 31-37.

Wolf, F. T. (1952). The production of indole acetic acid by Ustilago zeae, and its possible significance in tumour formation. Proc Natl Acad Sci U S A 38, 106.

Wösten, H. A., Bohlmann, R., Eckerskorn, C., Lottspeich, F., Bölker, M. \& Kahmann, R. (1996). A novel class of small amphipathic peptides affect aerial hyphal growth and surface hydrophobicity in Ustilago maydis. EMBO J 15, 4274-4281. 